\title{
CONTRIBUIÇÃO AO CONHECIMENTO DA BIOLOGIA DE CAPRELLA PENANTIS (LEACH) (CRUSTACEA, AMPHIPODA) DA ILHA DE ANHATOMIRIM, SANTA CATARINA
}

\author{
Josane Mittmann ${ }^{1}$ \\ Yara Maria Rauh Müller ${ }^{1}$
}

\begin{abstract}
Contribution to THE KNOWLedge of CAPRELla PENANTIS (LEACH) biology (Crustacea, Amphipoda) in Anhatomirim island, Santa Catarina. A total of 1257 caprellids, mostly females, were collected, from February to September 1991. The average sex-ratio was of 0,83 , with a range from 1,58 and 0,20 . Salinity ranged from $30 \%$ to $36 \%$; seawater and air temperature ranged from 26,00 to $17,66^{\circ} \mathrm{C}$ and 25,23 to $18,60^{\circ} \mathrm{C}$ respectively. Caprellid densities were higher during the cold months and males were the largest of the population. Males could be distributed in 12 length classes, while females in seven only.

KEY WORDS. Amphipoda, Caprella penantis, Anhatomirim, biology data
\end{abstract}

Caprella penantis (Leach 1814) (Amphipoda, Caprellidae) é muito comum em águas temperadas e tropicais (BYNUM 1978), sendo mais freqüente nos fitais do Rio Grande do Sul (AREzon \& Bond-BuCKuP 1991) e Paraná (DUTRA 1988).

Os caprelídeos são exclusivamente marinhos (NARCHI 1973; BYNUM 1978; BARNES 1984) e muito abundantes em estuários (CAINE 1983, 1991), em comunidades de "fouling", molhes e costões rochosos, ricos em moluscos (BYNUM 1978). Foram encontrados por DUTRA (1988) em todas as estações do ano, em temperaturas da água da superfície de $16,5^{\circ} \mathrm{C}$ (agosto) a $28^{\circ} \mathrm{C}$ (fevereiro) e salinidade de $26 \%$ (agosto) à 34\%o (abril), sendo que C. penantis apresenta altas densidades nos meses mais frios (DUTRA 1988).

Os caprelídeos são dificilmente reconhecíveis, pois sua forma, cor e postura os confundem com plantas e animais colôniais sobre os quais vivem. Segundo MCLAUGHLin (1980), o gênero Caprella (Lamarck 1801) apresenta um comprimento padrão de 1,00 a $32,00 \mathrm{~mm}$. Os machos são maiores que as fêmeas (NARCHI 1973; CAINE 1991) e em algumas espécies, como C. unica (Mayer 1890), chegam a ser duas vezes maiores, com valores de comprimento de $15,00 \mathrm{~mm}$ para os machos e de $8,00 \mathrm{~mm}$ para as fêmeas (BERRIL 1971). Em C. penantis, BYNUM (1978) registrou para as fêmeas um comprimento médio de $8,00 \mathrm{~mm}$.

De maneira geral para caprelídeos existe uma predominância de fêmeas na população. BYNUM (1978) encontrou para C. penantis e C. gorgonia (Laubitz \& Lewbel 1892) uma proporção sexual de 1:2.

1) Departamento de Biologia Celular, Embriologia e Genética, Universidade Federal de Santa Catarina. Caixa Postal 476, 88040-900 Florianópolis, Santa Catarina, Brasil. 
Dados biométricos, de ocorrência e da estrutura da população de C. penantis, são informações importantes para um melhor conhecimento das espécies que compõem a fauna dos ambientes continentais sulbrasileiros. Os Amphipoda e, dentre eles, os caprelídeos, têm um relevante papel na manutenção dos ecossistemas fitais (AREZON \& BOND-BUCKUP 1991).

\section{MATERIAL E MÉTODOS}

O material biológico foi obtido a partir de seis coletas realizadas no período de fevereiro a setembro de 1991 na Ilha de Anhatomirim, Santa Catarina ( $27^{\circ} 25^{\prime}$ S, $\left.48^{\circ} 33^{\prime} \mathrm{W}\right)$.

Os caprelídeos foram coletados em espinhéis de cultivo de mexilhões, removendo-se os indivíduos, juntamente com o substrato de hidróides e zoóides, até perfazer aproximadamente $500 \mathrm{ml}$.

Paralelamente à obtenção do material biológico coletou-se amostras de água do local para a determinação da salinidade, medida em partes por mil, com auxílio de refratômetro. Para as temperaturas do ar e da água utilizou-se as médias mensais obtidas no mesmo período pelo Laboratório de Mexilhões da Universidade Federal de Santa Catarina.

O material coletado foi passado em uma peneira de malha fina, fixado em formol $5 \%$ por aproximadamente 24 horas e conservado em álcool $70 \%$. A triagem foi feita retirando-se os caprelídeos do substrato e separando-os pelo sexo. A verificação do sexo foi feita segundo características morfológicas, apresentadas por NARCHI (1973); BYNUM (1978); MoORE \& Francis (1986) e CAINE (1991). No presente trabalho não foram considerados os indivíduos menores que $2,00 \mathrm{~mm}$, pela impossibilidade de determinação do sexo.

O comprimento total (extremidade distal do acúleo da testa até a extremidade distal do último segmento torácico) foi obtida utilizando-se uma Placa de Petri, com fundo em papel milimetrado e microscópio estereoscópico em aumento de 20X. A partir dessas medidas, os indivíduos foram classificados em classes de comprimento com $1,00 \mathrm{~mm}$ de intervalo.

\section{RESULTADOS}

Quando coletados, os indivíduos apresentavam colorações variadas, desde amarelo-creme até parda, dependendo do tipo de substrato em que estavam fixados.

Durante o período de estudo a salinidade variou de 30\% a 36\%, sendo que, para cada registro, não houve coincidência na concentração de sais dissolvidos. As temperaturas médias do ar e da água demonstraram a existência de períodos quentes e frios bem definidos, variando de $25,23^{\circ} \mathrm{C}$ a $18,6^{\circ} \mathrm{C}$ e de $26,99^{\circ} \mathrm{C}$ a $17,66^{\circ} \mathrm{C}$, respectivamente. A temperatura do ar e da água oscilaram em conjunto, sendo que a temperatura da água se manteve sempre superior a do ar (Fig. 1).

Foram capturados um total de 1.257 indivíduos, sendo 686 (54,57\%) fêmeas e $571(45,43 \%)$ machos, observando-se uma grande variação no número de indivíduos capturados em cada coleta. Em abril de 1991 foram coletados apenas 18 $(1,44 \%)$ indivíduos, em contraste com os 687 (54,87\%) coletados em julho (Fig. 2). 


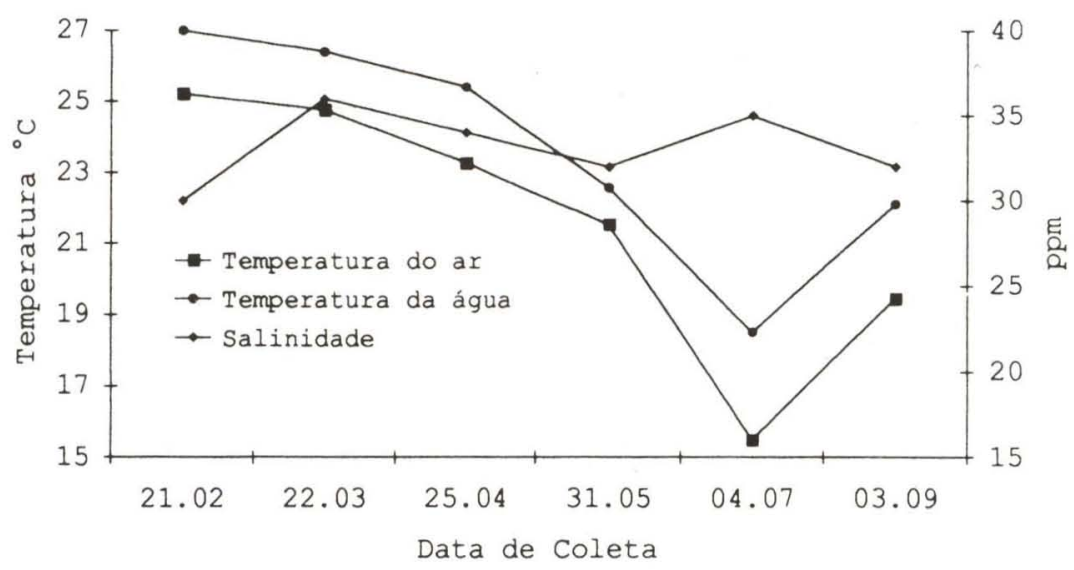

Fig. 1 Salinidade e medias mensais das temperaturas do ar e da água, registradas na Ilha de Anhatomirim, Santa Catarina, no periodo de fevereiro a setembro de 1991.

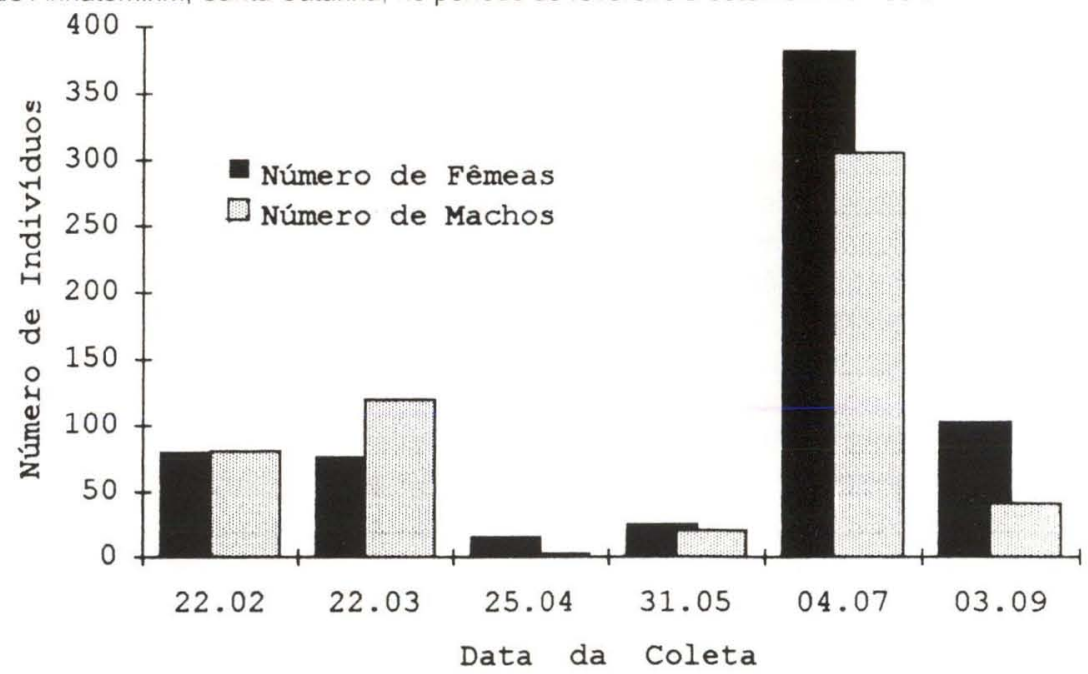

Fig. 2. Total de machos e fêmeas de Caprella penantis, coletados na Ilha de Anhatomirim, Santa Catarina, no período de fevereiro a setembro de 1991.

A razão sexual de todo o período de coleta foi de 0,83 e a razão sexual mensal variou de 1,58 a 0,20 . Os machos sempre foram encontrados em grande número, exceto em abril e maio, quando houve um decréscimo geral na população. A coleta em que a proporção sexual foi mais favorável às fêmeas foi em abril, coincidindo com o mês de menor captura de indivíduos (Tab. I).

As fềmeas medem, em média, $5,59 \mathrm{~mm}$ de comprimento e foram agrupadas em sete classes de comprimento. Os machos medem em torno de $7,57 \mathrm{~mm}$ e apresentam-se distribuídos em 12 classes de comprimento (Fig. 3). 
Tabela I. Número total de machos, de fêmeas e razão sexual da espécie Caprella penantis, coletada na llha de Anhatomirim, Santa Catarina, no periodo de fevereiro a setembro de 1991.

\begin{tabular}{lrcc}
\hline Data da coleta & Número de machos & Número de fêmeas & Razão sexual \\
\hline $22-I I-1991$ & 81 & 80 & 1,01 \\
$22-I I I-1991$ & 120 & 76 & 1,58 \\
$25-I V-1991$ & 3 & 15 & 0,20 \\
$31-V-1991$ & 21 & 25 & 0,84 \\
04-VII-1991 & 305 & 382 & 0,80 \\
03-IX-1991 & 41 & 108 & 0,38 \\
\hline Total & 571 & 686 & 0,83 \\
\hline
\end{tabular}

As fêmeas distribuem-se entre as classes I $(2,00-3,00 \mathrm{~mm})$ e VII $(8,00-$ $9,00 \mathrm{~mm}$ ), sendo que a maioria, $30 \%$ destas, encontram-se na classe V. Já os machos apresentam-se agrupados entre as classes II $(3,00-4,00 \mathrm{~mm})$ e XIII $(14,00-$ $15,00 \mathrm{~mm}$ ), apresentando uma distribuição bem mais ampla que as fêmeas, sendo que $16,00 \%$ encontram-se na classe III (Fig. 3).

\section{DISCUSSÃO}

Durante o período de estudo, houve flutuações na população de C. penantis. Os indivíduos apareceram em maiores densidades no inverno, onde foram registradas as menores médias mensais das temperaturas do ar e da água, dados que concordam com afirmações de DUTRA (1988). Valores diferentes foram encontrados por BYNUM (1978), em estações equivalentes, onde a população sofreu um declínio no inverno e um aumento na primavera. Segundo TAKEUCHI et al. (1990), a ocorrência de caprelídeos é influênciada pela forma de vida da alga ao qual estão associados e, BYNUM (1978) também demonstrou que existe uma correlação entre a presença do substrato e a densidade de $C$. penantis.

A razão sexual variou nos diferentes meses de coleta e na maioria das vezes foi favorável as fêmeas, dado comprovado pela razão sexual do período. Para a mesma espécie BYNUM (1978) registrou uma proporção sexual de 1:2, demonstrando uma predominância de fềmeas na população. Dados semelhantes também foram encontrados para outros anfípodas como Caprella gorgonia (BYNUM 1978), C. okadai (Arimoto 1930) e Orchestia gammarellus (Pallas 1902) (MOORE \& FRANCIS 1986).

Segundo CaINE (1991), o dimorfismo sexual é pronunciado em muitas espécies de caprelídeos, onde as fêmeas são menores que os machos em relação ao comprimento total, fato aqui confirmado para C. penantis. Observações semelhantes foram feitas por LAUBITZ \& MILLS (1978) para C. rinki (Stephensen 1917) e por TAKEUCHI et al. (1990) para C. okadai.

No presente estudo, a espécie C. penantis coletada na Ilha de Anhatomirim, Santa Catarina, apresenta uma distribuição mais ampla nas classes de comprimento do que a encontrada para a mesma espécie por DUTRA (1988) na Ilha do Mel, Pontal 
do Paraná, Paraná. Apesar das diferenças de amplitude, tanto nos nossos estudos como o relatado pelo autor acima citado, os machos são os maiores indivíduos na população, encontrando-se nas maiores classes de comprimento. Diferenças no tamanho do corpo são esperadas para a mesma espécie habitando diferentes ambientes, e segundo CAINE (1991), existe uma grande tendência de se acreditar que o comprimento do corpo decresce em latitudes menores.

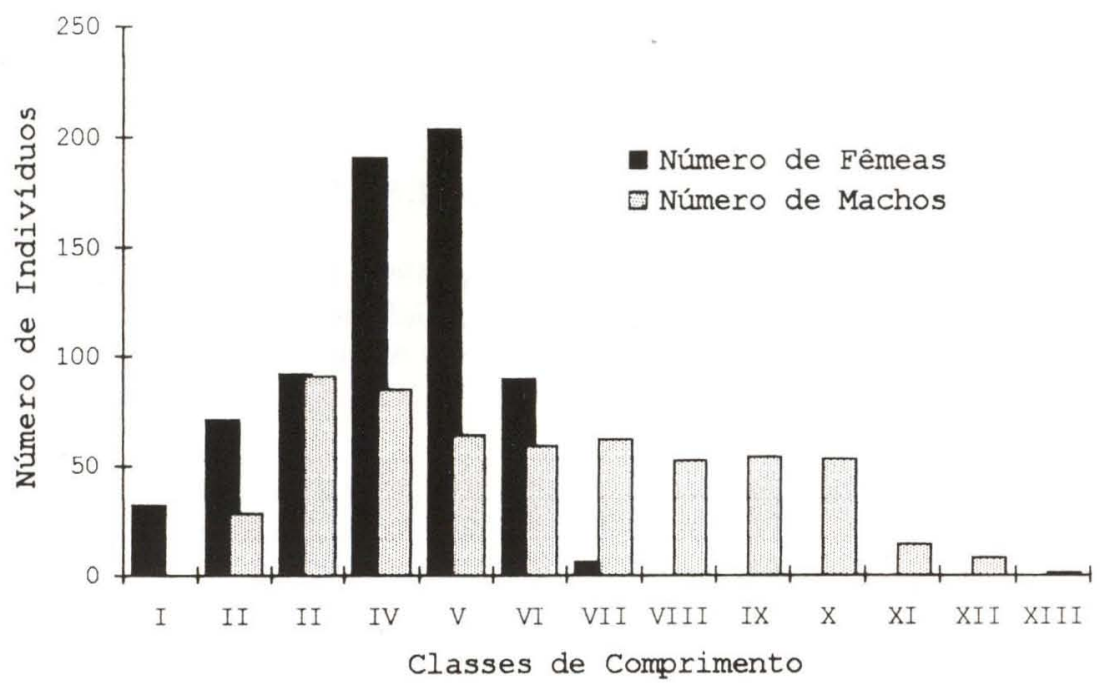

Fig. 3. Distribuição de frequências de machos e fêmeas de $C$. penantis, em classes de comprimento total, coletados na Ilha de Anhatomirim, Santa Catarina, no periodo de fevereiro a setembro de 1991.

FINANCIAMENTO. Desenvolvido com o apoio DAP/UFSC.

\section{REFERÊNCIAS BIBLIOGRÁFICAS}

Arezon, A. \& G. Bond-Buckup. 1991. Os Caprellidae do litoral do Rio Grande do Sul (Crustacea: Amphipoda). Comun. Mus. Ciênc. PUCRS, sér. zool., Porto Alegre, 4: 3-15.

BARNES, R.D. 1984. Zoologia de invertebrados. São Paulo, Editora Roca, 4ª ed., $1179 \mathrm{p}$.

Berril, M. 1971. The embryonic behavior of Caprella unica (Crustacea: Amphipoda). Can. Jour. Zool. 49: 499-504.

BYNUM, K.H. 1978. Reproductive biology of Caprella penantis Leach, 1814 (Amphipoda: Caprellidae) in North Carolina, U.S.A., Est. Coast. Mar. Sci. 7: 473-485.

CAINE, E.A. 1983. Community interactions of Caprella penantis Leach (Crustacea: Amphipoda) on sea whips. Jour. Crust. Biol. 3 (4): 497-504. 
1991. Reproductive behavior and sexual dimorphism of a Caprellid Amphipod. Jour. Crust. Biol. 11 (1): 56-63.

DUTRA, R.R.C. 1988. A fauna vágil do fital Pterocladia capillacea (Rhodophyta, Gelidiaceae) da Ilha do Mel, Paraná, Brasil. Rev. Brasil. Biol. 48: 589-605.

LAuBitz, D.R. \& E.L. MiLls. 1978. Deep-sea Amphipoda from the Western North Atlantic Ocean. Caprellidae. Can. Jour. Zool. 50 (4): 371-383.

McLaughlin, P.A. 1980. Comparative morphology of recent Crustacea. San Francisco, W.H. Freeman and Company, 177p.

MOORE. P.G. \& C.H. FrANCIS. 1986. Notes on breeding periodicity and sex ratio of Orchestia gammarellus (Pallas) (Crustacea: Amphipoda) at Millport, Scotland. Jour. Exp. Mar. Biol. Ecol. 95: 203-209.

NARCHI, W. 1973. Crustáceos - estudos práticos I. São Paulo, Polígono-EDUSP, $116 \mathrm{p}$.

Takeuchi, I; H. Yamakawa \& M. Fujiwara. 1990. Density of caprellid amphipods (Crustacea) inhabiting the red alga Gelidium amansii (Lamouroux) Lamouroux, with emphasis on Caprella okadai Arimoto. La Mer 28: 30-36.

Recebido em 13.IX.1995; aceito em 12.XII.1997. 